\title{
Ursachen und Wirkungen - Überlegungen zur Beibehaltung der Überhangmandate im neuen Wahlgesetz
}

\author{
Joachim Behnke
}

Nach langem Ringen verabschiedete der Deutsche Bundestag mit den Stimmen der CDU/ CSU-FDP-Regierungsmehrheit und gegen die Stimmen aller anderen Fraktionen am 29. September 2011 ein neues Wahlgesetz. Im Vorfeld dieser Entscheidung gab es eine umfassende Debatte, mit der es allerdings nicht gelang, einen Konsens über die Lösung herzustellen. Dies liegt wohl weniger an Unstimmigkeiten über die Einschätzungen der faktischen Gegebenheiten, denn in Hinsicht auf die „positiven“, also empirischen Eigenschaften verschiedener Wahlsystementwürfe gibt es unter den Forschern keine wesentlich unterschiedlichen Auffassungen. Hier hat die Wahlsystemforschung der letzten Jahre genügend Aufklärungsarbeit geleistet. ${ }^{1}$ Die Ursachen des mangelnden Konsenses liegen an anderer Stelle.

Es zählt zu den Absonderlichkeiten dieser Debatte, dass nicht einmal Einigkeit darüber besteht, welches Problem denn eigentlich zu lösen ist beziehungsweise worin die dem Parlament gestellte Aufgabe besteht. Den unmittelbaren Anlass bildete das Urteil des Bundesverfassungsgerichts zum negativen Stimmgewicht vom 3. Juli 2008. Darin bezeichnete das Gericht diesen Effekt als verfassungswidrig und gab dem Parlament den Auftrag, diesem Missstand abzuhelfen. Welche Art von Handlungsaufforderung sich aus diesem Urteil nun aber ergibt beziehungsweise ob sich relevante oder sogar zwingende Anforderungen auch aus anderen Urteilen ableiten lassen, ist strittig.

\section{Die „enge“ Auffassung des Auftrags}

Die enge Auffassung zum Auftrag des Bundestags, die von der Regierungskoalition vertreten wird, bezieht den Standpunkt, dass Handlungsbedarf des Parlaments nur in Hinsicht auf das Urteil des Verfassungsgerichts vom 3. Juli 2008 bestünde und dass aus diesem Urteil nur die Aufforderung zur Beseitigung des negativen Stimmgewichts abgeleitet werden könne. Eine Behandlung der Überhangmandate ist nicht vorgesehen. Ganz im Gegenteil:

1 Zu den aktuellen Vorschlägen vgl. unter anderem Friedrich Pukelsheim, Stellungnahme für die öffentliche Anhörung am 5. September 2011 zu den Gesetzentwürfen zur Änderung des BWahlG der Fraktionen CDU/CSU und FDP, SPD, Die Linke und Bündnis 90/Die Grünen, Ausschussdrucksache des Innenausschusses 17(4) 327 A, Berlin 2011; Joachim Behnke, Dies ist das Wahlsystem meines Missvergnügens - Eine Kritik des Entwurfs der CDU/CSU und FDP für eine Reform des Wahlgesetzes, Friedrichshafen 2011, http://www.zeppelin-university.de/deutsch/lehrstuehle/politikwissenschaft/CDU_Wahlreformentwurf.pdf (Abruf am 20. Januar 2012); Stephan Klecha, Zum Zusammenhang von Wahlrechtsreformen und Parteiensystem. Eine Debatte um Henne und Ei, in: ZPol, 58. Jg. (2011), H. 2, S. $324-345$. 
Die Opposition unterminiere sogar einen möglichen gemeinsamen Entwurf, indem sie sich auf das „sachfremde“2 Gebiet der Überhangmandate bezöge, so der Parlamentarische Geschäftsführer der Unionsfraktion, Peter Altmaier. Diese Behauptung muss allerdings zumindest mit einem Fragezeichen versehen werden. Man kann das Urteil womöglich so interpretieren, dass darin die Abschaffung der Überhangmandate weder explizit noch implizit gefordert ist, aber keineswegs ist daraus zu lesen, dass die Überhangmandate verfassungsrechtlich unbedenklich seien. Da der Gegenstand der Klage das negative Stimmgewicht und nicht die Überhangmandate waren, hat sich das Urteil zu diesen auch nicht direkt geäußert. Als Beleg für eine implizite „Absegnung“ der Überhangmandate wird darauf hingewiesen, dass das BVerfG bei der Nennung möglicher Lösungsvorschläge zur Beseitigung des negativen Stimmgewichts zum Beispiel die Trennung der Listen erwähnt, wobei die Überhangmandate ja unberührt blieben. ${ }^{3}$ Das Gericht weist aber ebenso auf Möglichkeiten hin, bei denen die Überhangmandate abgeschafft würden. In einem weiteren Urteil zu einer Wahlprüfungsbeschwerde vom 15. Januar 2009 befand das BVerfG unter Bezugnahme auf das Urteil vom 3. Juli 2008: „Es muss nicht entschieden werden, ob $₫ 6$ Abs. 5 Satz 2 und $\$ 7$ Abs. 3 Satz 2 in Verbindung mit $\$ 6$ Abs. 5 Satz 2 BWG insoweit gegen Art. 38 Abs. 1 Satz 1 GG verstoßen, als sie die Zuteilung von Überhangmandaten ohne Verrechnung oder Ausgleich zulassen. Denn das Bundesverfassungsgericht hat die vom Beschwerdeführer beanstandeten Regelungen aus einem anderen Grund für verfassungswidrig erklärt. Je nachdem für welche Lösung sich der Gesetzgeber entscheidet, ist die Frage der Verfassungsmäßigkeit der Mandatsverteilung dann auf der Grundlage des neuen Regelungskomplexes zu beurteilen. “4 Der Staatsrechtler Hans Meyer folgert daraus: „Über die Zulässigkeit von ,Überhangmandaten ohne Verrechnung oder Ausgleich` liegt nach dieser seiner letzten Entscheidung zur Sache jedenfalls noch keine gefestigte Rechtsprechung des Bundesverfassungsgerichts vor." 5

\section{Die weite Interpretation des Urteils}

Unter einer rein formalen Sichtweise kann den Vertretern der Koalition insofern in gewisser Weise zugestimmt werden, dass aus dem Urteil keine explizite Handlungsaufforderung, sich mit dem Problem der Überhangmandate zu befassen, folgt. Man kann vermutlich sogar so weit gehen anzunehmen, dass das BVerfG mit dem Urteil auch nicht die Absicht verfolgt hat, die Überhangmandate auf indirektem Wege zum Problem zu erklären. ${ }^{6}$ Allerdings ist dies in diesem Zusammenhang eher irrelevant. Während die Interpretation der

2 Peter Altmaier zitiert nach Anna Reimann, Urteil des Verfassungsgerichts, in: Spiegel Online vom 16. Juni 2011, http://www.spiegel.de/politik/deutschland/0,1518,768725,00.html (Abruf am 23. Januar 2012).

3 Vgl. BVerfG, 2 BvC 1/07 vom 3. Juli 2008, Abs. 142.

4 BVerfG, 2 BvC 4/04 vom 15. Januar 2009, Abs. 25 - 27.

5 Hans Meyer, Die Zukunft des Bundestagswahlrechts, Baden-Baden 2010, S. 51.

6 In dieser Hinsicht würde ich auch meine eigene, früher geäußerte Ansicht, das Urteil zum negativen Stimmgewicht sei als „trojanisches Pferd“ zur Abschaffung der Überhangmandate zu betrachten, revidieren (vgl. Joachim Behnke, Negatives Stimmgewicht, Erfolgswert und Überhangmandate - einige Anmerkungen, in: Kritische Vierteljahresschrift für Gesetzgebung und Rechtswissenschaft, 93. Jg. (2010), H. 1, S. 3-28). 
mit einer Handlung verfolgten Absicht einen wesentlichen Aspekt bei der Interpretation von Gesetzen darstellt, weil mit diesen ein bestimmter Wille durchgesetzt werden soll, geht es bei der Analyse eines Urteils „lediglich“ darum, ob die Urteilenden in ihrem Urteil und dessen Begründung auf nachvollziehbare Weise und in sich kohärent argumentiert haben. Es kommt dabei nicht darauf an, den „eigentlichen“ Willen der Urteilenden zu ergründen, sondern die dem Urteil zugrunde liegende Logik. Die wichtige Frage bei der Interpretation eines Verfassungsgerichtsurteils lautet nicht „Was hat das Gericht gewollt?“, sondern „Was hat das Gericht gemeint?". Entscheidend ist daher die Argumentstruktur der Karlsruher Richter bezüglich des negativen Stimmgewichts und deren Folgen. Die Gesetze der Logik sind unerbittlich und gelten unabhängig davon, ob man sie sich bewusst zu eigen macht beziehungsweise ihnen bewusst zu folgen versucht.

Es gibt (mindestens) zwei Argumentationsstränge, an denen gezeigt werden kann, welche unmittelbaren Konsequenzen sich aus der festgestellten Verfassungswidrigkeit des negativen Stimmgewichts für die Beurteilung der Überhangmandate ergeben. Der eine ist formaler Art und bezieht sich auf den normativen Maßstab, der andere ist pragmatischer Natur und bezieht sich auf den Kausalprozess, innerhalb dessen das negative Stimmgewicht und Überhangmandate entstehen. Ersterer ist sehr einfach und kurz. Bei ihrem Urteil haben die Richter den Maßstab der Erfolgswertgleichheit noch einmal deutlich enger und strenger gefasst als in der bisherigen Rechtsprechung. ${ }^{7}$ Wenn aber die relativ geringfügigen Verzerrungen der Erfolgswertgleichheit beim negativen Stimmgewicht für verfassungswidrig erklärt werden, gleichzeitig aber durch den Verweis auf die Lösungsmöglichkeiten implizit deutlich gemacht wird, dass Überhangmandate als nicht offensichtlich problematisch eingestuft werden, dann ist das Urteil in dieser Hinsicht „inkonsistent“8. Diese logische Inkohärenz wird noch dadurch verstärkt, dass das negative Stimmgewicht die verfassungsrechtlich unerwünschten Unterschiede der Erfolgswerte gar nicht einmal vergrößert, sondern sogar verkleinert, denn das „Mehr an Stimmen“, das zu einem „Weniger an Sitzen“ führt, bedeutet ja nichts anderes, als dass ein Mandat nun durch Zweitstimmen abgedeckt wird, das ansonsten als Überhangmandat angefallen wäre. ${ }^{9}$ Das mitunter vorgebrachte Gegenargument, dass auch ein „Weniger an Stimmen“ zu einem „Mehr an Sitzen“ und somit zu einer Vergrößerung der Unterschiede der Erfolgswerte führen könnte, ist lediglich die spiegelbildliche Formulierung des Effekts. Ausschlaggebend ist, dass der Wähler nur über die Entscheidung verfügt, seine Stimme einer Partei zu geben. Er kann sie ihr nicht entziehen beziehungsweise sie nichtig machen. Für den Wähler kann der unerwünschte Effekt des negativen Stimmgewichts daher nur darin bestehen, dass er durch seine Stimmabgabe seiner Partei geschadet hat. Die Erfolgswertgleichheit wird aber in diesem Fall besser angenähert. Die aus der Sicht des Wählers unerwünschte Wirkung seiner Stimmabgabe ist aus Sicht der normativen Beurteilung anhand des Maßstabs der Erfolgswertgleichheit zu begrüßen.

7 Vgl. Dieter Nohlen, Erfolgswertgleichheit als fixe Idee oder: Zurück zu Weimar? Zum Urteil des Bundesverfassungsgerichts über das Bundeswahlgesetz vom 3. Juli 2008, in: ZParl, 40. Jg. (2009), H. 1, S. 179 - 195; Gerald Roth, Negatives Stimmgewicht und Legitimationsdefizite des Parlaments, in: Neue Zeitschrift für Verwaltungsrecht (NVwZ), Bd. 11 (2008), S. 1199 - 1201.

8 Ebenda, S. 1200.

9 Vgl. Joachim Behnke, a.a.O. (Fn. 6). Im gleichen Sinne äußerte sich bei der Verhandlung über das negative Stimmgewicht auch der Prozessbevollmächtigte der CDU, Peter M. Huber (BVerfG, a.a.O. (Fn. 3), Absatz 74). 
Im Unterschied zum formalen Argument wird im „pragmatischen“ die Frage gestellt, wie eine sinnvolle Lösung des unerwünschten und ja unbestritten „absurden“ Effekts des negativen Stimmgewichts aussehen könnte. Diesen von den Überhangmandaten getrennt zu behandeln ist dabei nicht sinnvoll und auch nicht möglich, da beide „untrennbar“ miteinander zusammenhängen, wie das Bundesverfassungsgericht ebenfalls festgestellt hat. ${ }^{10}$ Dieser Zusammenhang wird von Vertretern von CDU/CSU in einer asymmetrischen Sichtweise allerdings gern so dargestellt, dass zwar das negative Stimmgewicht notwendig mit Überhangmandaten, aber nicht Überhangmandate notwendig mit dem Auftreten des negativen Stimmgewichts verbunden seien, wonach sich ihrer Meinung nach aus der Verfassungswidrigkeit des negativen Stimmgewichts nicht ableiten lasse, dass dies auch für Überhangmandate gelte.

Diese Argumentation muss aber als logisch ausgesprochen löchrig bezeichnet werden, denn im Kontext des geltenden Wahlsystems kann das negative Stimmgewicht, wie ja auch zugegeben wird, nicht ohne Überhangmandate auftreten. Ganz im Sinne einer klassischen Kausalitätstheorie müssen daher Überhangmandate als Ursache des Auftretens eines negativen Stimmgewichts betrachtet werden beziehungsweise im Sinne des INUS-Schemas von Mackie $^{11}$ als notwendiger, wenn auch isoliert nicht hinreichender Bestandteil eines Komplexes von Bedingungen, die zusammen genommen hinreichend, wenn auch nicht immer notwendig für das Entstehen der entsprechenden Wirkung sind. ${ }^{12}$ „Ursachen“, beziehungsweise das, was wir als Ursachen bezeichnen, sind immer eingebettet in einen Kontext von Zusatzbedingungen, ohne deren Vorliegen keine Wirkung zustande käme. Der Blitzeinschlag führt nur dann zu Feuer, wenn Sauerstoff vorhanden ist. Intuitiv würden wir aber in einem solchen Fall das herausgehobene Ereignis des Blitzeinschlags als „Ursache“ des Feuers betrachten.

Überhangmandate können in diesem Sinn vielleicht am besten mit flüssigem Nitroglyzerin verglichen werden. Es muss nicht explodieren, aber kleinste Erschütterungen reichen aus, um die Explosion hervorzurufen. Wir würden hier dennoch das Nitroglyzerin als maßgebliche Ursache für eine Explosion ansehen und nicht die Erschütterung, denn in der lebensweltlichen Erfahrung gilt Stolpern allgemein als harmlos, wenn man nicht gerade ein Reagenzglas mit flüssigem Nitroglyzerin bei sich trägt. ${ }^{13}$ Ähnlich wie Nitroglyzerin stellen Überhangmandate also ein potenzielles Risiko für das Entstehen des negativen Stimmgewichtseffekts dar; es bedarf dann nur noch des spezifischen Auslösers einer bestimmten Stimmenverteilung, um das Potenzial in den Effekt umzusetzen. Überhangmandate führen im Kontext des alten Wahlsystems notwendig dazu, dass ein negatives Stimmgewicht entstehen kann. Treten Überhangmandate in hinreichend vielen Bundesländern auf (dies be-

10 Vgl. BVerfGE 122, S. 311.

11 Vgl. John Leslie Mackie, The Cement of the Universe, Oxford 1980.

12 Dies wird korrekt so auch vom Abgeordneten Günter Krings der CDU erkannt: „Wie entsteht negatives Stimmgewicht? Es entsteht durch die Verbindung - das ist die erste Hauptursache von Landeslisten über ein Wahlsystem bei gleichzeitiger - das ist die zweite Hauptursache - Existenz von Überhangmandaten." (Plenarprotokoll 17/130, S. 15292.) Falsch hingegen ist die daraus geschlossene Schlussfolgerung, dass es demnach beliebig sei, welche der beiden Ursachen beseitigt wird.

13 Und wie Kenner der Filmgeschichte und Henri-Georges Clouzots „Lohn der Angst“ wissen, würde sich nur eine ganz und gar verlorene Existenz auf das Himmelfahrtskommando des Transports von Nitroglyzerin über unwegsame Straßen einlassen. 
ginnt schon bei zwei oder drei), so ist die Wahrscheinlichkeit sehr hoch, dass sich aus einem Wahlergebnis ein negativer Stimmgewichtseffekt konstruieren lässt.

Wenn aber der Effekt als verfassungswidrig angesehen wird, wie kann dann die Ursache, die zwangsläufig die Möglichkeit dieses Effekts nach sich zieht beziehungsweise die Wahrscheinlichkeit seines Auftretens dramatisch steigert, als unbedenklich eingestuft werden? Wenn jemand dafür verantwortlich gemacht wird, dass er in trunkenem Zustand einen Unfall verursacht hat, dann ja nicht wegen des Unfalls selbst - denn so etwas kann auch unter „harmlosen“ Umständen passieren, für die wir den Fahrer nicht verantwortlich machen würden -, sondern weil er durch den Alkoholkonsum die Wahrscheinlichkeit eines Unfalls beträchtlich erhöht hat und ihm dies überdies auch noch bewusst gewesen sein muss.

Umgekehrt gilt: Wenn Überhangmandate tatsächlich vollkommen unproblematisch sein sollten, weil sie eben gerade nicht dem Verhältnisausgleich unterliegen und aus diesem gewissermaßen bewusst herausgenommen werden - also im Sinne einer dem Proporz „vorgeschalteten Mehrheitswahl“, die diesen daher auch teilweise außer Kraft setzen kann -, wenn dies so sein sollte, dann ist nicht erkennbar, worin überhaupt ein Problem beim Effekt des negativen Stimmgewichts bestehen sollte, denn innerhalb des Proporzverfahrens gibt es keinen derartigen Effekt. Mehr Stimmen in Bundesland A im Vergleich zu B führen zu mehr Sitzen in A oder der gleichbleibenden Anzahl, in keinem Fall zu weniger Sitzen. Die Wirkung entsteht ja jetzt höchstens dadurch, dass das zusätzliche Proporzmandat in A nicht sichtbar wird, weil es lediglich zur Abdeckung eines bisherigen Überhangmandats führt und somit zu einer Umwandlung desselben in ein Proporzmandat, während gleichzeitig in B ein Proporzmandat verloren geht. Das negative Stimmgewicht entsteht durch die Interaktion eines in sich vollkommen sinnvoll, das heißt mit den Intentionen des Designs konform, ablaufenden Distributionseffekts und eines Unterdeckungseffekts ${ }^{14}$, der eben in der mangelnden Abdeckung von Direktmandaten durch Zweitstimmen besteht, also im Auftreten von Überhangmandaten. Wenn aber diese Unterdeckung grundsätzlich nicht zu bemängeln ist, dann gilt dies unabhängig von der Art und Weise, wie die Unterdeckung zustande kommt. Anders ausgedrückt: Wenn statische Ergebnisse jeweils für sich genommen unproblematisch sind, dann kann auch der Wechsel von einem solchen statischen Ergebnis zu einem anderen nicht problematisch sein. Gehen wir von zwei Szenarien aus: In Szenario 1 haben wir eine Zweitstimmenverteilung von $Z_{1}$ zwischen den Landeslisten einer Partei, in Szenario 2 eine Zweitstimmenverteilung $Z_{2}$. Die Verteilung der Direktmandate in den Ländern sei für die Partei in beiden Szenarien dieselbe, nämlich D. In Szenario 1 kommt es zu einer Verteilung von Überhangmandaten $\ddot{U}_{1}$, in Szenario 2 zu einer Verteilung $\ddot{U}_{2}$. Beide Szenarien stellen bezüglich des Wahlergebnisses realistische Zweitstimmenergebnisse dar. Wenn nun die Unterdeckung von Überhangmandaten durch Zweitstimmen grundsätzlich unproblematisch wäre, dann sind beide jeweils für sich genommen, vollkommen unproblematisch.

Nehmen wir an, Szenario 1 sei der Status quo, also das tatsächliche Ergebnis der letzten Wahl. Dann ist Szenario 2 eines der beliebten kontrafaktischen Szenarien in der Art: „Wenn das Ergebnis statt Szenario 1 das von Szenario 2 gewesen wäre, wäre es zu einem negativen Stimmgewichtseffekt gekommen." Des Weiteren nehmen wir an, dass beim (vor- 
gestellten) Übergang von Szenario $1 \mathrm{zu}$ Szenario 2 ein negatives Stimmgewicht entsteht, weil die Summe der Zweitstimmen in $Z_{1}$ kleiner als die von $Z_{2}$ ist, gleichzeitig aber im Szenario 1 mehr Überhangmandate auftreten. Der Unterschied der Zweitstimmensummen in den beiden Szenarien soll allerdings so gering sein, dass es auf der Oberverteilung zu derselben Anzahl von Proporzmandaten kommt.

Wenn nun aber Szenario 2 das tatsächliche Wahlergebnis gewesen wäre, dann wäre dieses für sich genommen genauso unproblematisch gewesen, wie es Szenario 1 für sich genommen ist. Der Übergang von einem möglichen Wahlergebnis, das für sich isoliert betrachtet unproblematisch ist, zu einem anderen möglichen Wahlergebnis, das für sich genommen ebenfalls unproblematisch ist, kann nicht unzulässig sein beziehungsweise unzulässige Elemente enthalten. Denn man kann wohl schlecht fordern, dass statt eines bestimmten Wahlergebnisses ein anderes nicht ebenso gut hätte zustande kommen dürfen. Der entscheidende Unterschied zwischen den beiden Szenarien besteht ja darin, dass sich die Anzahl der Überhangmandate verändert, obwohl sich weder die Verteilung der Direktmandate noch die Anzahl der Proporzmandate, die der Partei insgesamt zustehen, ändert. Wenn aber eine größere Anzahl von Überhangmandaten in einem der beiden Szenarien bei insgesamt gleichbleibender oder sogar leicht abnehmender Anzahl von Zweitstimmen grundsätzlich zulässig ist, dann ist der negative Stimmgewichtseffekt eben die logische Konsequenz der Zulässigkeit einer solchen Konstellation.

Zusammengefasst: Wenn Überhangmandate und ihre mangelnde Abdeckung durch Zweitstimmen grundsätzlich als unproblematisch anzusehen sind, ist es auch der negative Stimmgewichtseffekt, da er nur eine zwangsläufige Folge davon darstellt. Nach dem logischen Schlussschema des Modus tollens ${ }^{15}$ gilt dann aber: Ist der negative Stimmgewichtseffekt als verfassungswidrig und daher problematisch anzusehen, dann muss dies zwangsläufig auch für die Überhangmandate gelten.

Listenverbindung und Überhangmandate sind also keineswegs zwei miteinander verbundene „Hauptursachen“ für das negative Stimmgewicht, wie es der Abgeordnete Günter Krings ausdrückt, von denen wir ebenso gut die eine wie auch die andere heranziehen können, um die Wirkung zu beseitigen. Vielmehr wird im negativen Stimmgewicht der absurde Effekt enthüllt, dass Überhangmandate vollkommen losgelöst von der Zweitstimmenzahl entstehen, durch diese also nicht gedeckt werden müssen. Das, was uns am negativen Stimmgewicht stört, ist das, was es am Wirken der Überhangmandate sichtbar macht. Wird nun die Listenverbindung aufgelöst, wird lediglich die Sichtbarkeit des störenden Effekts beseitigt, aber nicht der Effekt selbst. Die interne Logik einer solchen Lösung ist vergleichbar mit einer, bei der man den kausalen Zusammenhang zwischen dem Trinken von gesüßtem Tee und Karies bei Kindern dadurch „unterbinden“wollte, indem man ihnen präventiv die Zähne zöge.

Es gibt noch ein anderes Argument, warum die Listenauflösung kein probates Heilmittel für die Beseitigung des negativen Stimmgewichts darstellt. Ihr Sinn wird häufig vor allem in der so genannten „Reststimmenverwertung“ gesehen. Dies ist aber eine unzutreffende Wahrnehmung. Wenn in den voneinander abgeschlossenen Wahlgebieten der Länder die

15 Wenn die so genannte Implikation $a \rightarrow b$ gilt, wenn also aus dem Auftreten von $a$ das Auftreten von $b$ folgt, dann kann aus dem Nichtauftreten von $b$ gefolgert werden, dass $a$ ebenfalls nicht aufgetreten sein kann. Wenn immer nachdem es geregnet hat, anschließend die Straße nass ist, die Straße im Moment aber trocken ist, dann kann es unmittelbar davor nicht geregnet haben. 


\begin{tabular}{|l|c|c|r|}
\hline \multicolumn{4}{|c|}{ Tabelle 1: Sitzverteilungen für zwei Szenarien, Stimmenzablen stehen in Klammern } \\
\hline & Partei A & Partei B & Partei C \\
\hline Szenario 1 & & & $1,24(1.240)$ \\
Land X & $3,51(3.510)$ & $5,25(5.250)$ & $3,09(3.090)$ \\
Land Y & $2,51(2.510)$ & $4,40(4.400)$ & $1,24(1.240)$ \\
\hline Szenario 2 & & $2,68(3.090)$ \\
Land X & $3,49(3.480)$ & $5,27(5.250)$ & \\
Land Y & $3,49(4.020)$ & $3,82(4.400)$ & \\
\hline \multicolumn{4}{|l}{ Quelle: Eigene Berechnungen. } \\
\hline
\end{tabular}

entsprechenden Sitzkontingente auf die einzelnen Listen der Parteien nach dem Sainte-Laguë-Verfahren verteilt werden, hat damit die Reststimmenverwertung eben schon stattgefunden, weil sie gewissermaßen im Verfahren implizit mit abgehandelt wird. Der tieferliegende Sinn der Listenverbindung liegt in der Minimierung des Effekts der mit der Reststimmenverwertung verbundenen Rundung der exakt proportionalen Sitzzahlen, die den Parteien aufgrund ihrer Stimmen zustehen, zu ganzen Zahlen. ${ }^{16}$ Bei einer Listenverbindung kommt es nur noch zu einer einzigen Rundung pro Partei, so dass es nicht zu der Addierung mehrerer Rundungseffekte kommen kann, die dann in der Summe besonders vorteilhaft oder ungünstig für bestimmte Parteien ausfallen können. Trennt man die Listen, kann es aufgrund dieser Rundungen zu einem Effekt des negativen Stimmgewichts kommen.

Nehmen wir an, in zwei Ländern $\mathrm{X}$ und $\mathrm{Y}$ würden jeweils zehn Mandate auf die drei Parteien A, B und C verteilt (siehe Tabelle 1). Bei einem spezifischen Szenario des Wahlergebnisses mit einer Verteilung der Zweitstimmen $Z_{1}$ kommt es zu einer Sitzverteilung, bei der zum Beispiel A in Land X 3,51 und in Land Y 2,51 Sitze erhält. Beide Zahlen werden aufgerundet, also kommt es insgesamt zu sieben Sitzen von A.

Nehmen wir an, dass A nun in X etwas weniger und in Y deutlich mehr Stimmen erhält, so dass sich, bei gleichbleibenden Stimmzahlen der anderen Parteien, die exakt proportionalen Sitzzahlen so verändern, dass A in X nun 3,49 und in Y 3,49 Sitze erhält. Obwohl also insgesamt die Stimmenzahl für A drastisch ansteigt, annähernd um ein Sitzäquivalent in Stimmen, erhält A nun insgesamt einen Sitz weniger, nämlich nur noch sechs Sitze. Dies entspricht aber genau der Definition des negativen Stimmgewichts seitens des Bundesverfassungsgerichts, nach der ein „Zugewinn von Zweitstimmen einer Partei zu einem Mandatsverlust bei genau dieser Partei (...) führen kann" ${ }^{17}$. Auch wenn die gängigen Beispiele zum negativen Stimmgewicht sich immer nur auf isolierte Zweitstimmengewinne auf einer Landesliste beziehen: In der Definition des Begriffs ist dies keineswegs angelegt; auch simultane Veränderungen der Zweitstimmen auf mehreren Listen sind zulässig, um den Effekt des negativen Stimmgewichts zu konstruieren. Getrennte Listen produzieren also

16 Die „Reststimmenverwertung“ im neuen Gesetz ist also einerseits eine Doppelung der Reststimmenverwertung, da diese ja schon zuvor in den Ländern stattgefunden hat; sie erfüllt aber gleichzeitig auch nicht den mit einer Listenverbindung erfüllten Zweck des Ausgleichs von addierten Rundungsfehlern, da ja nur Rundungseffekte in eine Richtung betrachtet werden. Diese „asymmetrische" Form der Reststimmenverwertung kann sogar zu einer weiteren Verschlechterung der Erfolgswertgleichheit führen.

17 BVerfG, a.a.O. (Fn. 3), Absatz 1. 
grundsätzlich die Möglichkeit der Entstehung von negativen Stimmgewichten. ${ }^{18}$ Dies würde selbst für getrennte Landeslisten mit festen Sitzkontingenten gelten, wenn man nicht die Restriktion, die Stimmengewinne müssten isoliert auf einer Landesliste stattfinden, anwendet. Werden die Sitzkontingente der Bundesländer an die Wählerstimmen gekoppelt, wie es das neue Wahlgesetz auf Vorschlag der Koalition vorsieht, dann kann der negative Stimmgewichtseffekt sogar zusätzlich noch bei isolierten Stimmgewinnen beziehungsweise -verlusten auf einer Liste entstehen. Die Listenverbindung kann also als eine Methode zur Vermeidung von negativen Stimmgewichtseffekten aufgrund von Rundungen betrachtet werden, auch wenn dies sicherlich beim Design so nicht beabsichtigt war.

Wenn die Verbindung von Landeslisten zusammen mit der Existenz von Überhangmandaten einen negativen Stimmgewichtseffekt hervorruft, sie aber ihrerseits einen negativen Stimmgewichtseffekt anderen Ursprungs verhindert, dann ist offensichtlich die Wahl, welche der beiden „Hauptursachen“ des negativen Stimmgewichts zur Beseitigung desselben berücksichtigt werden sollte, keineswegs beliebig. Vielmehr ist jenes Instrument zu bevorzugen, das bei seiner Anwendung nicht das zu beseitigende Problem auf andere Weise wieder von Neuem produziert.

Richtig ist, dass der Effekt des negativen Stimmgewichts, wenn er lediglich aufgrund von Rundungen auftritt, nicht antizipierbar ist und somit nicht bewusst manipulativ eingesetzt werden kann. Aber das BVerfG hat nicht die Instrumentalisierung des Effekts für verfassungswidrig erklärt, sondern sein Auftreten an sich. Die Instrumentalisierbarkeit fügt dem Ganzen sicherlich Brisanz hinzu, hat aber nichts mit der „Widersinnigkeit“ der Wirkung selbst zu tun, die ja den grundlegenden Stein des Anstoßes darstellt. Wenn aber die Instrumentalisierbarkeit im alten System tatsächlich als wichtiges Problem erkannt werden sollte, ist darauf hinzuweisen, dass diese ja nur im Zusammenhang mit Überhangmandaten auftrat, also wenn „risikolos“ in Ländern, bei denen die Entstehung von Überhangmandaten zu vermuten war, eine Unterdeckung der dort anfallenden Direktmandate betrieben werden konnte. Das Problem der instrumentellen Ausnutzbarkeit des Effekts des negativen Stimmgewichts, wie zum Beispiel im Falle der Nachwahl in Dresden bei der Bundestagswahl $2005^{19}$, ist also ebenfalls ein Problem der Überhangmandate. Diese Form der Instrumentalisierbarkeit aber wird durch das neue Gesetz ebenfalls nicht beseitigt, da es die Überhangmandate intakt lässt.

Überhangmandate sind also mit Sicherheit alles andere als eine „sachfremde“ Erwägung im Zusammenhang mit der Reform des Wahlgesetzes. Es ist sehr naheliegend, wenn nicht sogar zwingend notwendig, sich mit ihnen auseinanderzusetzen.

\section{Der über das BVerfG-Urteil von 2008 hinausgehende Auftrag, insoweit er sich aus anderen Entscheidungen ergibt}

Der Handlungsauftrag an den Bundestag ergibt sich aber nicht nur aus dem Urteil von 2008 zum negativen Stimmgewicht. Auch aus anderen Entscheidungen des BVerfG lässt sich die Notwendigkeit einer Wahlsystemreform ableiten.

18 Vgl. hierzu genauer Joachim Behnke, a.a.O. (Fn. 1) und Stephan Klecha, a.a.O. (Fn. 1).

19 Vgl. Joachim Behnke, Strategisches Wählen bei der Nachwahl in Dresden zur Bundestagswahl 2005, in: PVS, 49. Jg. (2008), H. 4, S. $695-720$. 
Die Koalition hat - wie übrigens auch alle anderen Parteien in ihren Entwürfen - in dem von ihr konzipierten neuen Wahlgesetz eine Passage eingeführt, die das Problem der so genannten „Berliner Zweitstimmen“ lösen sollte. Hierbei handelt es sich um den Umstand, dass 2002 die Zweitstimmen der Wähler, die mit der Erststimme eine der beiden erfolgreichen Wahlkreiskandidatinnen der PDS, Gesine Lötzsch und Petra Pau, gewählt hatten, gewertet wurden und in die Proporzverrechnung eingingen, obwohl die PDS nicht zuteilungsberechtigt war und demnach von vornherein die Abgeltung der Direktmandate mit den Mandaten, die der Partei aufgrund der Zweitstimmen zugeteilt werden, unmöglich war. Eine Inkonsistenz der Gesetzeslage besteht insofern, als Zweitstimmen für erfolgreiche unabhängige Kandidaten nicht weiter berücksichtigt werden, und zwar weil sie gar nicht abgegolten werden können.

Wähler, die 2002 mit der Erststimme eine der erfolgreichen Berliner PDS-Kandidatinnen und mit der Zweitstimme zum Beispiel SPD oder Grüne gewählt hatten, besaßen demnach ein doppeltes Stimmgewicht. Der Sinn des Verhältnisausgleichs und der Nichtberücksichtigung der Zweitstimmen von Wählern, die mit der Erststimme erfolgreiche unabhängige Kandidaten gewählt haben, besteht genau darin, ein solches doppeltes Stimmgewicht grundsätzlich zu vermeiden. Das Bundesverfassungsgericht hat diese Inkonsistenz mehrfach bemängelt und eine entsprechende Korrektur gefordert. ${ }^{20}$ Wenn aber das doppelte Stimmgewicht im Fall der Berliner Zweitstimmen ein Problem darstellt, dann muss dies offensichtlich auch für den Fall der Überhangmandate gelten, denn auch hier kommt es ja zu einem doppelten Stimmgewicht. ${ }^{21}$ Ansonsten läge die von Gerald Roth in Bezug auf das 2008er Urteil bemängelte Inkonsistenz der Rechtsprechung vor. ${ }^{22}$ In der Anhörung des Innenausschusses des Bundestages zum neuen Wahlgesetz stellte der von der Regierungskoalition benannte Sachverständige Heinrich Lange die Behauptung auf, man könne die Berliner Zweitstimmenproblematik mit den Überhangmandaten nicht vergleichen. Im ersten Fall ginge es nämlich um die Fünfprozentklausel, im zweiten Fall aber um ein „ganz anderes Strukturproblem“, da die Überhangmandate aus der "Verbindung der beiden Wahlsysteme“ entstünden. ${ }^{23}$ Dieser Auffassung schloss sich auch ein zweiter von der Koalition benannter Sachverständiger, Bernd Grzeszick, an, der Überhangmandate als Konsequenz einer „Grundsystementscheidung" des Gesetzgebers sah, in der dieser weitgehend frei sei. ${ }^{24}$ Es fällt schwer, dieses Argument nachzuvollziehen. Man kann wohl davon ausgehen, dass für die Beurteilung des Bundesverfassungsgerichts nicht die Form des zugrunde liegenden „Strukturproblems" entscheidend war, sondern die Einhaltung des Maßstabs der Erfolgswertgleichheit. An diesem müssen sich sowohl Fünfprozenthürde und Berliner Zweitstimmen als auch die Überhangmandate messen lassen. Der Aussage des Sachverständigen Friedrich Pukelsheim: „Das doppelte Stimmgewicht bei den Überhangswählern ist dasselbe wie das doppelte Stimmgewicht bei den ,Berliner Zweitstimmen “'25 ist daher eindeutig zuzustimmen. Die Freiheit des Gesetzgebers bei der Systementscheidung könnte nur dann als hinreichender Grund für die Hinnahme einer Verzerrung des Erfolgswertes angesehen werden,

20 Vgl. BVerfGE 79, S. 161; BVerfGE 122, S. 304.

21 Vgl. ausführlich hierzu Hans Meyer, a.a.O. (Fn. 5), S. 51, S. $55 \mathrm{ff}$.

22 Vgl. Gerald Roth, a.a.O. (Fn. 7), S. 1200.

23 Innenausschuss, Protokoll Nr. 17/48, S. 28.

24 Ebenda, S. 42.

25 Ebenda, S. 39. 
wenn diese unterschiedlichen Erfolgswerte bewusst gewollt gewesen wären oder wenn sie eine zwar ungewollte, aber unvermeidbare, also notwendige Folge der Entscheidung für das personalisierte Verhältniswahlsystem wären. Man kann aber ausschließen, dass das System bewusst auf eine Erfolgswertdifferenzierung ausgelegt ist. Gerade im Urteil von 2008 wird dies noch einmal ausdrücklich klargestellt: Das BVerfG geht weiterhin vom „Grundcharakter der Verhältniswahl“26 aus und konstatiert, „dass dem Gesetzgeber bei der Ordnung des Wahlrechts nur ein eng bemessener Spielraum für Differenzierungen bleibt. (...) Differenzierungen bedürfen zu ihrer Rechtfertigung stets eines besonderen, sachlich legitimierten, ,zwingenden' Grundes"27. Wären die Erfolgswertunterschiede durch das Design des Systems beabsichtigt und fiele dies in den Gestaltungsspielraum des Gesetzgebers, bedürften sie keiner Rechtfertigung. Differenzierungen im Wahlrecht können aber sehr wohl „durch Gründe gerechtfertigt werden, die durch die Verfassung legitimiert und von einem Gewicht sind, das der Wahlgleichheit die Waage halten kann"28. Im Falle der Fünfprozenthürde wird dieser Grund in der Aufrechterhaltung der Funktionsfähigkeit des Parlaments gesehen. Überhangmandate aber sind eben nicht zwingend die Folge der Systementscheidung für eine personalisierte Verhältniswahl. Sie können beseitigt werden, ohne in das Element der Personenwahl einzugreifen. Folglich können sie nicht als hinzunehmendes Übel betrachtet werden, um das größere Gut der personalisierten Verhältniswahl zu erhalten. ${ }^{29}$

Auch das umfangreiche Urteil zu den Überhangmandaten von 1997 weist auf mehrere Umstände hin, unter denen diese problematisch werden könnten: „Der Grundcharakter der Wahl als Verhältniswahl lässt eine Differenzierung des Gewichts der für die Parteien abgegebenen Stimmen nicht unbeschränkt zu. (...) Werden diese Grenzen überschritten, weil sich Verhältnisse einstellen, unter denen Überhangmandate von Wahl zu Wahl regelmäßig in größerer Zahl anfallen, entfernt sich das Wahlverfahren von den Grundentscheidungen des Gesetzes. Aus diesen Anforderungen an das Wahlverfahren kann sich einerseits eine Schranke für den Gestaltungsspielraum des Gesetzgebers wie andererseits aufgrund der Änderung der tatsächlichen Verhältnisse auch ein Handlungsauftrag ergeben." 30

Es liegt nahe zu vermuten, dass das Gericht davon ausging, dass die 1994 angefallenen 16 Überhangmandate, die Anlass der Klage waren und eine vollkommen neue Größenordnung des Auftretens von Überhangmandaten darstellten, schon als eine solche "größere“ Anzahl anzusehen waren. Diese Zahl ist aber in vier der letzten fünf Bundestagswahlen erreicht oder sogar überschritten worden. Es kann daher mit einiger Plausibilität auch eine gewisse Regelmäßigkeit des Auftretens der Überhangmandate in „größerer Zahl“ angenommen werden - mit der Folge, dass sich ein Handlungsauftrag an das Parlament ergeben würde.

26 BVerfG, a.a.O. (Fn. 3), Absatz 96.

27 Ebenda, Absatz $97 \mathrm{f}$.

28 Ebenda, Absatz 98.

29 Vgl. Joachim Behnke, Überhangmandate und negatives Stimmgewicht: Zweimannwahlkreise und andere Lösungsvorschläge, in: ZParl, 41. Jg. (2010), H. 1, 247 - 260; Friedrich Pukelsheim / Matthias Rossi, Wahlsystem nahe Optionen zur Vermeidung negativer Stimmgewichte, in: Juristen Zeitung, 65. Jg. (2010), H. 19, S. $922-929$.

30 BVerfGE 95, S. 335, S. 365 f.; vgl. auch die kritische Diskussion in Hans Meyer, a.a.O. (Fn. 5), S. $52 \mathrm{ff}$. 


\section{4. Überhangmandate sind ein Problem - ob sie als verfassungswidrig erkannt werden oder nicht}

Selbst wenn man die Hypothese akzeptierte, dass der Gesetzgeber durch das Verfassungsgericht nicht eindeutig gezwungen ist, gegen den Missstand der Überhangmandate vorzugehen, hieße das ja keineswegs, dass er nicht tätig werden darf beziehungsweise nicht sollte. Denn man mag es drehen und wenden, wie man will, die Überhangmandate sind in jedem Fall - unabhängig von ihrem verfassungsrechtlichen Status - ein schwerwiegender Makel im derzeitigen Wahlsystem.

Es gibt keinerlei überzeugendes Argument, das für eine bewusste Erhaltung der Überhangmandate ins Feld geführt werden könnte. ${ }^{31}$ Überhangmandate sind bestenfalls ein hinzunehmendes Übel, niemals ein positiv zu wertendes Element des Wahlsystems, zu dessen Erhalt aktiv Bestrebungen unternommen werden sollten. Dies ist, wie zuvor erläutert, auch im Wesentlichen die Haltung des Bundesverfassungsgerichts. Überhangmandate sind kein bewusst im Design des Wahlsystems angelegter Effekt; nur dann könnten sie im Sinne der Freiheit der Systemwahl begründet werden. Sie sind eine nicht gewollte Nebenwirkung des Systems.

Es gab und gibt immer wieder die Tendenz, die Überhangmandate als zwar nicht gewollten, aber nichtsdestoweniger durchaus begrüßenswerten Nebeneffekt zu betrachten. Früher wurde hier im Sinne eines so genannten „Prämienmodells“ argumentiert, nach dem die Überhangmandate als Bonus für jene Parteien anfielen, die besonders attraktive Kandidaten in den Wahlkreisen aufgestellt hatten. Dem widerspricht allerdings schon allein die Tatsache, dass der dramatische Anstieg der Überhangmandate nicht dadurch zustande gekommen ist, dass bestimmte Parteien in letzter Zeit besonders erfolgreich waren, sondern dass sie besonders schwach geworden sind; jene die den Großteil der Direktmandate erhalten, bekommen immer weniger Zweitstimmen. Warum aber dieser Popularitätsabfall mit zusätzlichen Sitzen belohnt werden sollte, ist nicht nachvollziehbar.

Pappi und Herrmann ${ }^{32}$ argumentieren gar wider den verfassungsrechtlichen Strich. Sie sehen nicht die Überhangmandate als Bonus an, sondern das doppelte Stimmgewicht von strategisch splittenden Wählern, die so zur Entstehung von Überhangmandaten beitragen. Das doppelte Stimmgewicht wird diesen Wählern sozusagen als Belohnung für ihre strategische Koordinationsleistung gewährt. Da diese Koordinationsleistung vor allem von Wählern jener Parteien vollbracht werde, die sich vor der Wahl auf eine klare Koalitionsaussage festgelegt haben, führe dies zu höheren Gewinnchancen von Bündnissen, die stabile Verhältnisse versprechen. Auch dieses Argument ist - ganz unabhängig von seiner Bedenklichkeit hinsichtlich des verfassungsrechtlichen Grundsatzes der Wahlgleichheit - nicht schlüssig, denn der Wille zum Bilden einer Koalition von zwei Parteien mit jeweils 20 Prozent der Stimmen kann genau so groß sein wie der einer Koalition aus einer 35 Prozent- und einer Fünfprozentpartei, dennoch wird nur letztere in der Lage sein, sich effektiv zu koordi-

31 Vgl. Joachim Behnke, Überhangmandate. Ein (behebbarer) Makel im institutionellen Design des Wahlsystems, in: ZPol, 13. Jg. (2003), H. 3, S. 1235 - 1269; ders. / Florian Grotz, Das Wahlsystem zwischen normativer Begründung, empirischer Evidenz und politischen Interessen, in: ZParl, 42. Jg. (2011), H. 2, S. $419-425$.

32 Vgl. Franz Urban Pappi / Michael Herrmann, Überhangmandate ohne negatives Stimmgewicht: Machbarkeit, Wirkungen, Beurteilung, in: ZParl, 41. Jg. (2010), H. 2, S. 260 - 278. 
nieren und grundsätzlich auch diejenige sein, die in den Genuss von Überhangmandaten gelangt. ${ }^{33}$ Die entscheidende Frage lautet daher: Gibt es eine normativ begründete Rechtfertigung dafür, dass eine Koalition, die die größte Partei beinhaltet, einen Sitzbonus erhält, der die Regierungsbildung erleichtert? Sind Bündnisse, die die größte Partei beinhalten, in irgendeiner Form besonders ausgezeichnet, so dass sie es besonders „verdienen“, die Regierung zu bilden? In der Tat gibt es Wahlsysteme, die eine solche „Konzentration“, verbunden mit einem Vorteil für große und einem Nachteil für kleine Parteien, bewusst vorsehen.

Der klassische Fall ist natürlich das Mehrheitswahlsystem wie in Großbritannien, in dem diese Disproportionalität so groß ausfällt, dass in der Regel die größte Partei allein die Regierung bilden kann. Dieser Effekt der Bildung so genannter „manufactured majorities" 34 ist aber gewollt, genau darin liegt der Sinn eines solchen Designs. Und in der Tat, wenn man der Ansicht ist, dass der Sieger eine Einparteiregierung bilden sollte, dann sollte der Sieger auch die größte Partei sein. ${ }^{35}$ Im Verhältniswahlsystem ist aber die Siegerkoalition diejenige, die in der Lage ist, eine Mehrheit im Parlament zu erringen. Im Falle einer halbwegs proportionalen Umrechnung von Stimmen in Sitze bedeutet dies zwangsläufig, dass die Regierungskoalition mehr Stimmen erringen konnte als die Opposition. Dies ist die normative Rechtfertigung für den Gewinn der Regierung im Verhältniswahlsystem. Erhielte hier eine Koalition die Mehrheit an Sitzen, die weniger Stimmen hinter sich hat als die übrigen Parteien, widerspräche dies der grundlegenden Intention des Designs eines Verhältniswahlsystems. ${ }^{36}$ Ein pragmatisches Argument für die disproportionale Konzentration im Design kann nur dann gegeben sein, wenn man zwar prinzipiell eine Koalitionsregierung haben möchte, aber gleichzeitig davon ausgeht, dass diese politisch nur unter Einschluss der größten Partei möglich und nur dann eine gewisse Stabilität garantiert ist. Von diesen Verhältnissen ist die Bundesrepublik aber weit entfernt. Dass diese disproportionale Konzentration auch nicht vom Gesetzgeber gewollt ist, zeigt sich ebenfalls in der Ablösung des Verrechnungsverfahrens d'Hondt durch Hare-Niemeyer seit der Bundestagswahl 1987. Also selbst die - im Vergleich zu den Überhangmandaten - relativ leichte Bevorzugung großer Parteien wurde aus Gründen der Fairness und der Chancengleichheit abgeschafft. Diese Prinzipien werden von allen Parteien anerkannt. ${ }^{37}$

33 Genauer Joachim Behnke / Florian Grotz, a.a.O. (Fn. 30).

34 Douglas W. Rae, The Political Consequences of Electoral Laws, New Haven 1967, S.74 f.

35 Tritt genau dieser gewünschte Designeffekt nicht ein, das heißt kommt es dazu, dass die zweitstärkste Partei eine Mehrheit an Sitzen erhält, spricht man dementsprechend von „perverse outcomes" (Paul Mitchell, The United Kingdom. Plurality Rule under Siege, in: Michael Gallagher I ders. (Hrsg.), The Politics of Electoral Systems, Oxford 2005, S. 157 - 184, S. 173).

36 Analog zum britischen Beispiel in Fußnote 34 müsste man dann auch hier von „perverse outcomes“, also „umgedrehten“ oder „widersinnigen“ Mehrheiten sprechen.

37 Vgl. Plenarprotokoll 10/120, S. 8937 ff. In dieser Debatte gibt es mehrere explizite Hinweise darauf, dass diese Änderung aus Gerechtigkeitsgründen durchgeführt wird, so vom CDU-Abgeordneten Franz Heinrich Krey (S. 8938) und vom FDP-Abgeordneten Torsten Wolfgramm auch mit dem Hinweis auf die Erfolgswertgleichheit (S. 8941). Vorbehalte gab es von dem SPD-Abgeordneten Günter Kiehm gegen die Zusatzsitzkorrektur, die ermöglichen soll, dass die Stimmenmehrheit einer Partei garantiert eine Mehrheit von Sitzen erhält (S. 8940). Das Gesetz wurde dennoch als Ganzes mit einer Enthaltung und nur wenigen Gegenstimmen angenommen. Allerdings ist hinzuzufügen, dass die Änderung des Berechnungsverfahrens nur ein Teil der Gesetzesänderung war, der andere bezog sich auf die Ausdehnung des Wahlrechts für im Ausland lebende Deutsche. 
Es kann also - auch aus Sicht der Parteien - keine positive Rechtfertigung der Überhangmandate selbst geben. Die Streitfrage ist bestenfalls, ob Überhangmandate ein zwar unerwünschtes, aber hinnehmbares, da nicht vermeidbares Übel darstellen, oder ob sie ohne allzu große schmerzliche Nebenwirkungen sehr wohl abgeschafft oder neutralisiert werden können. Der erste Schritt für eine solche fruchtbare Debatte aber bestünde darin, dass vor allem CDU und CSU offen bekennen, dass auch sie Überhangmandate für ein Übel halten, anstatt schamhaft an ihnen auf eine Weise festzuhalten, die suggeriert, dass sie ja durchaus wünschenswerte Folgen produzierten.

\section{Die bewusste Produktion von Überhangmandaten widerspricht demokratietheoretischen Prinzipien}

Die „wünschenswerten Folgen“ der Überhangmandate lassen sich offensichtlich nur als spezifische Machtinteressen einzelner Parteien formulieren, taugen aber eben nicht als normative Rechtfertigung. Der offene Verweis auf Machtinteressen wäre aber nicht nur kein Argument, sondern darüber hinaus eine moralische Bankrotterklärung der Parteien, sich bewusst Vorteile zu verschaffen, von denen sie wissen, dass sie darauf keinen gerechtfertigten Anspruch erheben können.

Dass genau dies aber der Fall ist, drängt sich allein durch die Beobachtung auf, dass die Befürworter der Überhangmandate immer deren Profiteure waren. Meist versuchen sie gar nicht mehr, positiv zu argumentierten, sondern begnügen sich damit, den Gegnern zu unterstellen, lediglich die eigenen Interessen zu verfolgen beziehungsweise verfolgt zu haben. So lautete der durchaus berechtigte Vorwurf von CDU und FDP an die SPD, diese hätte die Überhangmandate nicht abschaffen wollen, als diese zu ihrem Vorteil waren. ${ }^{38}$ In der Tat ist es kein Ruhmesblatt für die Glaubwürdigkeit der Sozialdemokraten, dass sie es in den sieben Jahren ihrer Regierungstätigkeit nicht für nötig befunden haben, den Missstand abzuschaffen, den sie selbst zuvor noch durch eine Verfassungsklage beseitigen lassen wollten; und in der Tat ist es für die SPD opportun, nun, da die Überhangmandate ihr schaden, gegen sie zu sein. Doch diese Vorwürfe der Scheinheiligkeit gehen am ethischen Kern des Problems vorbei. Es ist vollkommen unerheblich, was die Motive für die derzeitige Position der SPD in Bezug auf die Überhangmandate sein mögen. Selbst wenn die Partei nur der opportunistische Trittbrettfahrer auf dem Ticket der Fairness- und Gerechtigkeitserfordernisse sein sollte, die die Überhangmandate eindeutig verbieten, so sind diese Erfordernisse eben ganz unabhängig davon die maßgeblichen Gegenargumente bei der Beurteilung der Überhangmandate.

Es gehört zu den Regeln einer pragmatischen Ethik, dass derjenige, dessen Handlungen mit eigennützigen Motiven erklärt werden können, unter besonderem Rechtfertigungsdruck steht. Umso mehr muss er sein Tun auch ethisch begründen können, wenn diese eigennützigen Motive mit dem Erlangen politischer Macht verknüpft sind. Wenn nun diejenigen, die in der aktuellen Situation eigene Interessen haben, an den Überhangmandaten festzuhalten, die moralische Glaubwürdigkeit der Gegner damit zu unterminieren versuchen, dass diese ihrerseits aus denselben eigennützigen Motiven nichts gegen die Überhangmandate getan hätten, solange sie zu ihrem Vorteil waren, so kehrt dieser Vorwurf wie ein Bumerang zurück und beschädigt die moralische Glaubwürdigkeit der Ankläger. 
Dieses Spiel wird auch umgekehrt betrieben: Die SPD weist ihrerseits darauf hin, dass Volker Kauder, Vorsitzender der Unionsfraktion, auch schon einmal gegen die Überhangmandate war, als sie der CDU schadeten. ${ }^{39}$ Das Entscheidende ist, dass die vielfachen Positionswechsel der Parteien offensichtlich vor allem mit ihren machtpolitischen Interessen zu erklären sind und nicht mit einer erkennbaren Änderung ihrer normativen Bewertung der Überhangmandate.

Es mag auf den ersten Blick banal erscheinen, darauf hinzuweisen, dass Politiker Interessen haben, die sie durchzusetzen versuchen. Wenn diese Interessen aber die Verteilung der Macht selbst betreffen, tangiert diese „Banalität“ unmittelbar das Prinzip der Legitimation in einer repräsentativen Demokratie. Versteht man dies - wie es heutzutage in der Demokratietheorie üblich ist - im Sinne von John Lockes Treuhänderschaft-Beziehung so, dass die Regierung durch die Wähler beauftragt worden ist, die Interessen der Bürger zu vertreten, so wäre es offensichtlich zu kurz gesprungen, die Legitimation allein durch den Akt der Wahl selbst schon gewährleistet zu sehen. Die Wahl stellt den Ermächtigungsvorgang lediglich in einem formalen Sinne dar. Sie dient im Falle der repräsentativen Demokratie vor allem der Auswahl der Agenten, die im Interesse des Prinzipals Volk handeln sollen, wobei die Gesamtheit der Agenten in einer pluralistischen Demokratie als Ausdruck der individuellen Interessenvielfalt einerseits und des Gemeinwohls andererseits zu verstehen ist. Der formale Akt der Delegation entbindet den Agenten in keiner Weise davon, weiterhin verpflichtet zu sein, die Interessen der Bevölkerung in diesem Sinne nach bestem Wissen und Gewissen zu vertreten. ${ }^{40}$ Der Vertretene hat keinen Anspruch, wenn ihm Schaden durch die Handlungen des Bevollmächtigten entsteht, solange dieser im „guten Glauben“ davon ausgegangen ist, im Interesse des Mandanten zu agieren. Wenn aber dieser Anspruch des Handelns im guten Glauben nicht erfüllt wird, entfällt die Grundlage des Ermächtigungsverhältnisses selbst. Überhangmandate aber sind eindeutig vor allem im Interesse der Repräsentanten, nicht der Repräsentierten, jedenfalls nicht ihrer Mehrheit. Der CDU-Abgeordnete, der für ein Gesetz stimmt, das von Hans Meyer treffend als „Überhangsicherungsgesetz " bezeichnet worden ist ${ }^{41}$, verletzt die Interessen (und auch Rechte) all jener Bürger eklatant, die womöglich mit ihrer Stimme bei der nächsten Wahl nicht mehr zu einer Mehrheit von Sitzen beitragen können, die sie ohne das neue Wahlgesetz gehabt hätten. Diese Bürger mögen nicht seine Wähler sein, er ist ihnen aber nicht weniger verpflichtet, denn jeder Abgeordnete ist der Repräsentant des ganzen Volkes. Wenn es gute Gründe gäbe, zu vermuten, dass diese Entscheidung nicht bloß aufgrund von Ignoranz und nicht im „guten Glauben“ in einer bestimmten Weise getroffen worden ist, sondern zur Verfolgung eigener Interessen, dann stellte dies eine schwerwiegende Verletzung der treuhänderischen Beziehung dar. Eine solche Entscheidung gegen die Mehrheit der Bürger kann auch nicht im Sinne des freien Mandats, wie es zum Beispiel von Edmund Burke verstanden wurde, damit gerechtfertigt werden, dass es sogar geradezu die Pflicht des Delegierten sein kann, sich gegen den Willen seiner Wähler zu stellen, wenn er aufgrund seiner höheren Kompetenz in einer Frage zu einer anderen Beurteilung kommt. Doch Burke spricht hier

39 Vgl. ebenda, S. 15297.

40 Dies würde höchstens für eine Form des Repräsentationsverständnisses im Sinne von Thomas Hobbes gelten, bei dem die Bürger ihre Rechte an den Leviathan abtreten, sich also ihrer Rechte entäußern (vgl. Jean Hampton, Political Philosophy, Boulder 1997, S. 49 ff.).

41 Drucksache des Innenausschusses 17 (4) 327 B, S. 1. 
von „opinions“ der Bürger und nicht ,interests“ “2, und die Nichtberücksichtigung der „opinions" wird im Namen der „eigentlichen" Interessen der Wähler, auch wenn sie ihnen nicht bewusst sein mögen, oder des Gemeinwohls gerechtfertigt. Bei der Frage des Stimmgewichts aber geht es unmittelbar um die Interessen der Bürger an einer fairen Einflussnahme bei der Auswahl der Mandatsträger. Eine Stimme des Abgeordneten beim Gesetzgebungsprozess gegen die Interessen der Mehrheit der Bürger kann daher logischerweise nicht im „eigentlichen“, aber nicht erkannten Interesse der Bürger liegen.

\section{Das Selbstverständnis des Gesetzgebers}

Ein Gesetzgeber, der seine Selbstbeschreibung so vornimmt, einen Handlungsbedarf nur dann zu sehen, wenn er durch verfassungsrechtliche Rechtsprechung dazu genötigt wird, und nicht dann, wenn es ein erkennbar schlechtes Gesetz durch ein besseres zu ersetzen gilt, erklärt sich selbst für überflüssig. Ein solches Parlament hätte es nicht verdient, gewählt zu werden und würde wohl auch nicht gewählt, nicht zu den Kosten, die es den Bürgern auferlegt. Ein selbstbewusstes Parlament stellt seine Daseinsberechtigung unter Beweis, indem es aktiv an der Entwicklung von Gesetzen wirkt, die die Wahrung der Interessen des Souveräns, also des Volks, im bestmöglichen Sinn gewährleisten. Überhangmandate aber sind nicht nur ein Problem in verfassungsrechtlicher Hinsicht, sie widersprechen auch den einfachen Gerechtigkeits- und Fairnesserfordernissen. Zudem stellen sie eines der für den Wähler unverständlichsten und mit Sicherheit am wenigsten verstandenen Elemente des Wahlsystems dar. Der „worst case“, eine Regierungsmehrheit, die nur mit Hilfe von Überhangmandaten zustande kommt, hätte mit ernsthaften Legitimationsproblemen zu kämpfen, weil der Bevölkerung nicht vermittelbar wäre, wie sie zustande gekommen ist. All dies sollte für den Gesetzgeber hinreichender Grund zum Handeln sein - Verfassungsgerichtsurteile hin oder her.

42 Edmund Burke, Speech to the Electors of Bristol, in: Francis Canavan (Hrsg.), Select Works of Edmund Burke: Miscellaneous Writings, Indianapolis 1999, S. 3 - 13, S. 10. 